\title{
Hydrolysis of Cellulose in Supercritical Water: Quantum Simulation
}

\author{
Taketo Oku¹, Mitsuhiro Matsumoto ${ }^{1}$ \\ ${ }^{1}$ Graduate School of Engineering, Kyoto University \\ Kyoto-Daigaku-Katsura, Kyoto 615-8540, Japan \\ oku.taketo.57n@st.kyoto-u.ac.jp; matsumoto@kues.kyoto-u.ac.jp
}

\begin{abstract}
Among several fabrication processes of cellulose nanofibers (CNF), we focus on the hydrolysis of cellulose in supercritical water. In order to analyse the detailed chemical reaction, a series of quantum molecular dynamics simulation were performed based on the density functional theory coupled with the tight binding model (DFTB). After locating the vapor-liquid critical point of the DFTB water system, we explored the hydrolysis reaction of cellulose using a simplified system consisting of a cellobiose and 100 water molecules. We observed cleavage of a $\beta$-glycosidic bond at a high temperature $(1000 \mathrm{~K})$. The charge analysis suggests that the oxygen atom at the cleavage site gives an electron to a water molecule approaching with sufficiently large velocity.
\end{abstract}

Keywords: cellulose nanofiber, hydrolysis, supercritical water, MD simulation, DFTB

\section{Introduction}

Cellulose nanofiber (CNF) is fairly new nanomaterial made from various type of cellulose. They have recently attracted much attention in many fields [1], such as electronic device, medical material, and food additives. One of the significant features is its high aspect ratio; a typical width is $4-100 \mathrm{~nm}$ while the length is often more than $5 \mu \mathrm{m}$. It is so light and tough that many applications as structural material are proposed, such as automobile bodies. Cellulose is a renewable resource since it is mainly made from wood pulp.

To fabricate CNF from conventional cellulose resources (e.g., wood pulp and grass), some treatment is required to smash macromolecules of cellulose. Currently two methods are major: (i) Chemical treatment [2], which uses hydrolysis reaction with strong acid or catalysts. This is not environment-friendly nor cost-effective. (ii) Mechanical approach [3], in which the pulp is mechanically smashed or ground under to high shear force. It generally gives large dispersion of fiber size. Less popular treatment is the hydrolysis using supercritical water [4], which is environment-friendly and has a possibility of precise size control, but its detailed mechanism is not fully understood.

In this study, we focus on the cellulose hydrolysis in supercritical water. To analyse the chemical reaction in atomic scale, we executed a series of molecular dynamics (MD) simulations with quantum effects taken into account as the density functional tight binding (DFTB) model.

\section{Methods}

To investigate the chemical reaction (hydrolysis), we have utilized the DFTB+ software [5] to perform MD simulations. A self-consistent charge (SCC) DFTB calculations are adopted with the third order correction [6]. The 3ob-3-1 parameter set is used [7], which is known to be well applicable to a wide range of organic and bio molecules. Use of appropriate hydrogen bond correction is relevant to our investigation, and we adopted a damping method for modification of short range potential energy $U[6]$, as

$$
\begin{gathered}
U=\frac{1}{2} \sum \Delta q_{a} \Delta q_{b} \gamma_{a b} \\
\gamma_{a b}=\frac{1}{r_{a b}}-S\left(r_{a b}, U_{a}, U_{b}\right) \times \exp \left[-\left(\frac{U_{a}+U_{b}}{2}\right)^{\zeta} r_{a b}^{2}\right]
\end{gathered}
$$

where $r_{a b}$ is the distance between atom $a$ and $b, \Delta q_{i}$ is the electric charge on atom $i, U_{i}$ is the Hubbard parameter of atom $i$, and $S$ is a short-ranged damping function [6]. The time step for the MD calculation is $0.25 \mathrm{fs}$. 


\section{Evaluation of Critical Point}

Before starting the main simulations, we have to determine the critical point of the model water. Using 100 water molecules confined in a cubic simulation cell of various size with periodic boundary conditions, we performed the DFTB MD simulations under constant temperature condition (Fig. 1); three temperature conditions were chosen, 400, 650 and 800 $\mathrm{K}$.

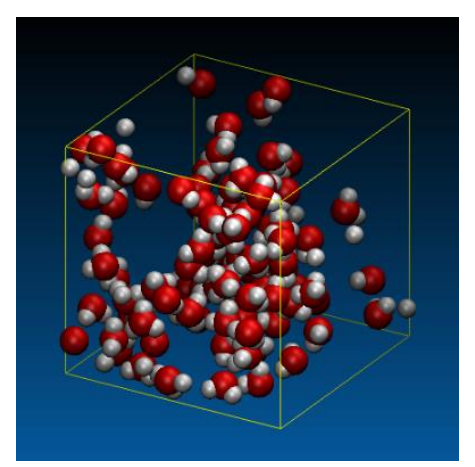

Fig. 1: Example of snapshot for pure water system with density $0.513 \mathrm{~g} / \mathrm{cm}^{3}\left(18 \times 18 \times 18 \mathrm{~A}^{3}\right)$.

After equilibrating the system, the mean pressure was evaluated. The obtained $p$ - $V$ curve is shown in Fig. 2, which suggests that the liquid-vapor critical point of this model water exists around $\left(T_{c}, V_{c}\right)=\left(650 \mathrm{~K}, 80 \mathrm{~A}^{3}\right) ; 80 \mathrm{~A}^{3}$ per molecule corresponds to the density $\rho_{c} \cong 0.374 \mathrm{~g} / \mathrm{cm}^{3}$. A reported experimental value for water is $\left(647 \mathrm{~K}, 0.322 \mathrm{~g} / \mathrm{cm}^{3}\right.$, $22.1 \mathrm{MPa}$ ) [8], and the agreement is reasonable.

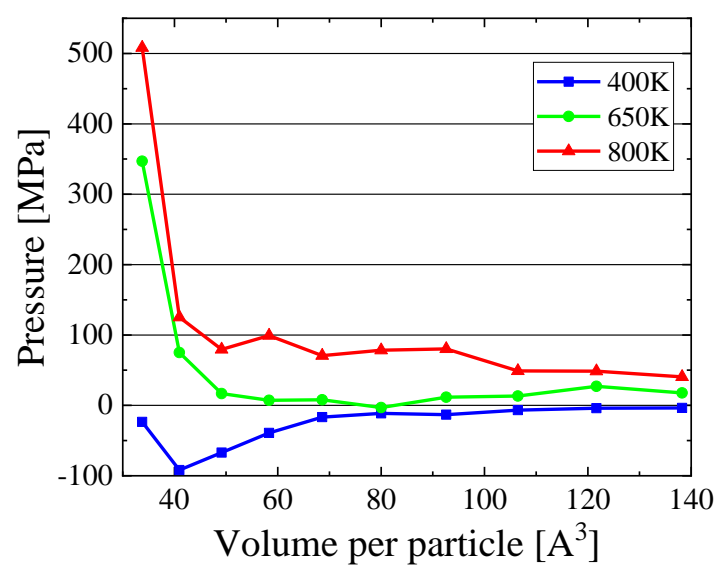

Fig. 2: $p-V$ relation for the pure water system.

To look at the fluid structure near the critical point, the oxygen-oxygen radial distribution function is calculated at $V=80 \mathrm{~A}^{3}$. Based on the results shown in Fig. 3, clustering is suggested at $400 \mathrm{~K}$, while uniform gas phase is realized at $800 \mathrm{~K}$. Thus the state in between $(650 \mathrm{~K})$ is close to the critical point. Based on these results, we performed the main simulation of hydrolysis at $T \geq 650 \mathrm{~K}$. 


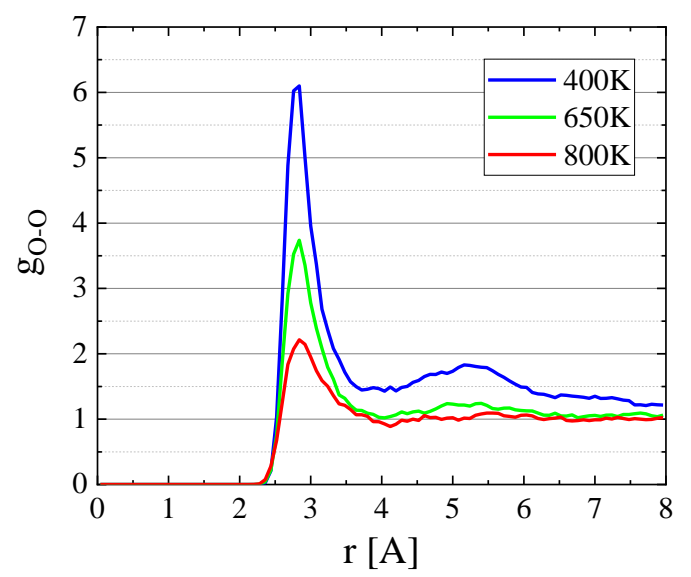

Fig. 3: O-O radial distribution function of fluid water with $V=80 \mathrm{~A}^{3}$ per molecule.

\section{Hydrolysis of Cellobiose}

We performed DFTB-MD simulations for the hydrolysis of cellulose in water at supercritical states. To reduce the computational resource, a minimum unit of cellulose, i.e., a cellobiose molecule, was targeted, which is surrounded by 100 water molecules, as shown in Fig. 4. The size of the simulation cell is $23.107 \times 23.107 \times 9.58 \mathrm{~A}^{3}$ with periodic boundary conditions. 50 water molecules are arranged at each side of the cellobiose. Constant temperature MD simulations were carried out at 650,800 and $1000 \mathrm{~K}$.

At low temperatures of 650 and $800 \mathrm{~K}$, no cleavage of $\beta$-glycosidic bond was observed during our observation $(50,000$ steps $=12.5 \mathrm{ps}$ for each temperature condition with several different initial configurations). At $1000 \mathrm{~K}$, the bond breaks after 3000 steps, as shown in Fig. 5.

The bond cleavage seems a rare event, and we need many samplings to investigate the statistical properties of the cleavage process. As a preliminary analysis, we have evaluated the electric change (Mulliken charge) on the oxygen atom in the bond. As shown in Fig. 5, the electron around the reaction site is lost before the breakage, similar to the hydrolysis in the strong acid [9].

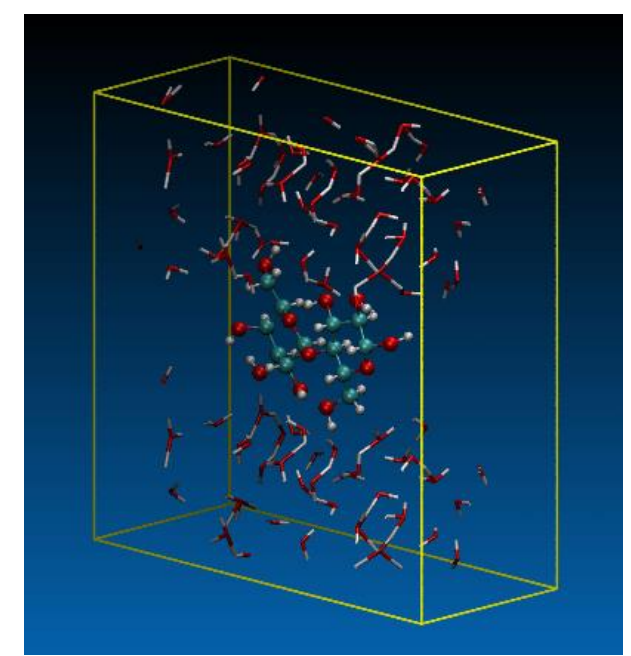

Fig. 4: Snapshot of a cellobiose surrounded by 100 water molecules. 


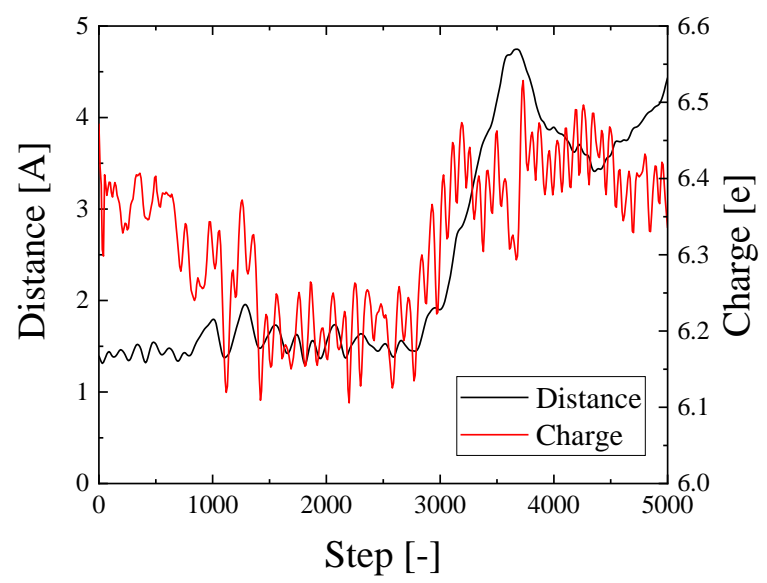

Fig. 5: Change of $\beta$-glycosidic bond length and electric charge on the bonding oxygen atom.

\section{Conclusion}

We examined a model cellulose in fluid water with quantum molecular dynamics simulation and found the of hydrolysis in supercritical conditions. However, the $\beta$-glycosidic bond is so strong that it requires large kinetic energy (high temperature, such as $1000 \mathrm{~K}$ ) of water molecules. The bond cleavage is initiated by the loss of electron on the bonding oxygen atom.

\section{References}

[1] D. Klemm, F. Kramer, S. Moritz, T. Lindstrom, M. Ankerfors, D. Gray and A. Dorris, "Nanocelluloses: A new family of nature-based materials," Angew. Chem. Int. Ed., vol 50, pp. 5438-5466, 2011.

[2] T. Saito, Y. Nishiyama, J. L. Putaux, M. Vignon and A. Isogai, "Homogeneous suspensions of individualized microfibrills from TEMPO-catalyzed oxidation of native cellulose," Biomolecules, vol. 7, no. 6, pp. 1687-1691, 2006.

[3] K. Abe, S. Iwamoto and H. Yano, "Obtaining cellulose nanofibers with a uniform width $15 \mathrm{~nm}$ from wood," Biomacromolecules, vol. 8, no. 10, pp. 3276-3278, 2007.

[4] M. Sasaki, Z. Fang, Y. Fukushima, T. Adschiri and K. Arai, "Dissolution and hydrolysis of cellulose in subcritical and supercritical water," Ind. Eng. Chem. Res., vol. 39, no. 8, pp. 2883-2890, 2000.

[5] B. Aradi, B. Hourahine and Th. Frauenheim, "DFTB+, a sparse matrix-based implementation of the DFTB method," $J$. Phys. Chem. A, vol. 111, no. 26, pp. 5678-5684, 2007.

[6] M. Gaus, Q. Cui and M. Elstner, "DFTB3: Extension of the self-consistent-charge density-functional tight-binding method (SCC-DFTB)," J. Chem. Theory Comput., vol. 7, pp. 931-948, 2011.

[7] M. Gaus, A. Goez and M. Elstner, "Parametrization and benchmark of DFTB3 for organic molecules," J. Chem. Theory Comput., vol. 9, pp. 338-354, 2013.

[8] W. Wagner and A. Pruß, "IAPWS formulation 1995 for the thermodynamic properties of ordinary water substance for general and scientific use,” J. Phys. Chem. Ref. Data, vol. 31, no. 2, pp. 387-535, 2002.

[9] C. Loerbroks, A. Heimermann and W. Thiel, "Solvents effects on the mechanism of cellulose hydrolysis: A QM/MM study," J. Comput. Chem., vol. 36, pp. 1114-1123, 2015. 